\title{
High-flow nasal cannula oxygen therapy versus conventional oxygen therapy in patients after planned extubation: a systematic review and meta-analysis
}

\author{
Youfeng Zhu ${ }^{1+} \mathbb{D}$, Haiyan Yin ${ }^{1+}$, Rui Zhang ${ }^{1}$, Xiaoling Ye ${ }^{1}$ and Jianrui Wei ${ }^{2^{*}}$
}

\begin{abstract}
Background: The effect of high-flow nasal cannula (HFNC) therapy in patients after planned extubation remains inconclusive. We aimed to perform a rigorous and comprehensive systematic meta-analysis to robustly quantify the benefits of HFNC for patients after planned extubation by investigating postextubation respiratory failure and other outcomes.

Method: We searched MEDLINE, EMBASE, Web of Science, and the Cochrane Library from inception to August 2018. Two researchers screened studies and collected the data independently. Randomized controlled trials (RCTs) and crossover studies were included. The main outcome was postextubation respiratory failure.

Results: Ten studies (seven RCTs and three crossover studies; HFNC group: 856 patients; Conventional oxygen therapy (COT) group: 852 patients) were included. Compared with COT, HFNC may significantly reduce postextubation respiratory failure $(\mathrm{RR}, 0.61 ; 95 \% \mathrm{Cl}, 0.41,0.92 ; z=2.38 ; P=0.02)$ and respiratory rates (standardized mean differences $(\mathrm{SMD}),-0.70 ; 95 \% \mathrm{Cl},-1.16,-0.25 ; z=3.03 ; P=0.002)$ and increase $\mathrm{PaO}_{2}(\mathrm{SMD}, 0.30 ; 95 \% \mathrm{Cl}, 0.04$, $0.56 ; z=2.23 ; P=0.03)$. There were no significant differences in reintubation rate, length of ICU and hospital stay, comfort score, $\mathrm{PaCO}_{2}$, mortality in ICU and hospital, and severe adverse events between HFNC and COT group.

Conclusions: Our meta-analysis demonstrated that compared with COT, HFNC may significantly reduce postextubation respiratory failure and respiratory rates, increase $\mathrm{PaO}_{2}$, and be safely administered in patients after planned extubation. Further large-scale, multicenter studies are needed to confirm our results.
\end{abstract}

Keywords: High-flow nasal cannula, Oxygen therapy, Mechanical ventilation, Postextubation

\section{Background}

Mechanical ventilation is associated with significant complications that are time-dependent in nature, with a longer duration of intubation resulting in a higher incidence of complications, such as ventilator-associated pneumonia (VAP) and increased mortality [1].

\footnotetext{
* Correspondence: Jianruiw@163.com

${ }^{\dagger}$ Youfeng Zhu and Haiyan Yin have equal contributions and are co-first authors.

${ }^{2}$ Department of Cardiology, Guangzhou Red Cross Hospital, Medical College, Jinan University, Tongfuzhong Road No. 396, Guangzhou 510220, Guangdong, China

Full list of author information is available at the end of the article
}

Extubation is beneficial in that it decreases the risk for VAP, eliminates the work of breathing imposed by the endotracheal tube, and improves patient comfort [2].

However, after extubation, functional residual capacity which was maintained by positive end-expiratory pressure (PEEP) in invasive ventilation duration might decrease rapidly, leading to hypoxemia and extubation failure. Extubation failure, which is often defined as the need for reintubation within $24-72 \mathrm{~h}$ after a planned extubation, is frequent, with rates of $10-20 \%$ [3-5]. Furthermore, extubation failure is associated with an overall increase in the duration of mechanical ventilation, a greater need for tracheostomy, higher medical costs, and an increased mortality [5-7]. 
Conventional oxygen therapy (COT) is the main supportive treatment administered to patients after planned extubation and has conventionally been delivered using nasal prongs, cannula or masks. However, the maximal oxygen flow rates that these devices can deliver are limited. The maximal oxygen flow rate delivered by COT is only $15 \mathrm{~L} / \mathrm{min}$, which is far lower than the demands of postextubation patients with acute respiratory failure [8]. Therefore, ambient air dilutes the supplied oxygen, and finally, the fraction of inspired oxygen $\left(\mathrm{FiO}_{2}\right)$ is significantly reduced in the alveoli. Furthermore, with oxygen delivered by COT, it is difficult to meet the requirements of heating and humidification in these patients [9]..

High-flow nasal cannula (HFNC) can supply a mixture of air and oxygen via a heated and humidified circuit at a very high flow. It can provide almost pure oxygen with a $\mathrm{FiO}_{2}$ of approximately $100 \%$ and a maximal flow rate up to $60 \mathrm{~L} / \mathrm{min}$ [8]. The use of a HFNC may generate a positive airway pressure, ameliorate oxygenation and dyspnea, reduce the respiratory rate and work of breathing, and improve comfort $[8,10-16]$.

However, the effect of HFNC therapy in patients after planned extubation remains inconclusive. Some studies demonstrate that HFNC after extubation can reduce the requirement for escalation of the respiratory support, result in better oxygenation $[17,18]$, and be associated with better comfort and a lower reintubation rate [12]. However, in the study by Corley and colleague, HFNC therapy did not show an improvement in respiratory function in patients after planned extubation with a body mass index (BMI) $\geq 30 \mathrm{~kg} /$ $\mathrm{m}^{2}[19]$.

Therefore, we aimed to perform a rigorous and comprehensive systematic meta-analysis to robustly quantify the benefits of HFNC for patients after planned extubation by investigating postextubation respiratory failure and other outcomes.

\section{Methods}

We performed this study in accordance with the Preferred Reporting Items for Systematic Reviews and Meta-Analyses (PRISMA) statement [20] and guidelines described in the Cochrane Handbook for Systematic Reviews of Interventions [21].

\section{Study selection criteria Types of studies}

Randomized controlled studies and crossover studies comparing HFNC and COT in the treatment of patients after planned extubation were included.

The exclusion criteria were case reports, animal studies, preclinical studies, or patients younger than 18 years.

\section{Types of participants}

Adult patients, who had undergone mechanical ventilation in the hospital or intensive care unit (ICU) and had planned extubation, were involved.

\section{Types of interventions}

Patients in the control group and intervention group received COT and HFNC therapy after extubation, respectively.

\section{Types of outcome measures}

Our primary outcome was postextubation respiratory failure, and the secondary outcomes included the following variables: reintubation, mortality in ICU and hospital, length of ICU and hospital stay, comfort score, respiratory rate, partial pressure of arterial oxygen $\left(\mathrm{PaO}_{2}\right)$, partial pressure of arterial carbon dioxide $\left(\mathrm{PaCO}_{2}\right), \mathrm{PaO}_{2} / \mathrm{FiO}_{2}$, and severe adverse events. Postextubation respiratory failure was defined as $\mathrm{PaO}_{2} / \mathrm{FiO}_{2}<$ $300 \mathrm{mmHg}$, hypoxemia $\left(\mathrm{PaO}_{2}<60 \mathrm{mmHg}\right.$ or $\mathrm{SpO}_{2}<$ $90 \%$ with $\left.\mathrm{FiO}_{2} \geq 0.5\right)$, respiratory acidosis $(\mathrm{pH}<7.35$ and $\mathrm{PaCO}_{2}>45 \mathrm{mmHg}$ ), signs of respiratory muscle fatigue and/or tachypnea $>35$ breaths/min, low level of consciousness, or agitation during treatment period [22, 23]. Severe adverse events were defined as respiratory pauses with loss of consciousness, severe unstable hemodynamics, and cardiac or respiratory arrest.

\section{Data sources and search strategy}

We searched EMBASE, MEDLINE, Web of Science, and the Cochrane Library from inception to August 2018. We also reviewed the references of relevant articles to avoid missing any studies. The details of the search strategy are shown in the Additional file 1: Appendix 1 . There were no limitations on gender, patient sample size, or language.

\section{Data extraction}

Two researchers (XLY and RZ) independently and repetitively screened titles and abstracts to evaluate the potential studies. Disagreements were resolved by consensus or by discussion with a third author (JRW). For the included studies, a full-text review was performed. Detailed study information, interventions, controls, and outcomes were extracted using a standardized data extraction form.

\section{Quality assessment}

The qualities of the included randomized studies were assessed by modified Jadad scores, with scores of $1-3$ and 4-7 judged as low and high quality, respectively. Furthermore, the included studies were evaluated for the risk of bias according to the methods described in the Cochrane Handbook [21]. 


\section{Statistical analysis}

Our meta-analysis was performed on an intention-to-treat basis and involved all patients who were assigned to any study group. Data were obtained by direct extraction or by indirect calculation. For studies that reported data with cartograms, we extracted data with DigitizeIt software (Braunschweig, Germany).

For binary outcomes, we calculated the risk ratios (RRs) and 95\% confidence intervals (CIs). For continuous outcomes, the standardized mean differences (SMDs) and 95\% CI were calculated. We graphically displayed the outcomes by forest plots and visually inspected the potential publication bias with a funnel plot.

We used DerSimonian-Laird random effects models for pooling outcomes. The Mantel-Haenszel model was used for assessment of heterogeneity, with $P<0.05$ and $I^{2}>50 \%$ indicating significant heterogeneity, and $I^{2}>$ $25 \%$ indicating moderate heterogeneity.

We performed prespecified subgroup analyses for the postextubation respiratory outcomes, including study types, HFNC duration, HFNC flow, severity of patients, hypercapnic or not, and post cardiac surgery or not.

We also used trial sequential analysis to estimate the reliability of our meta-analysis by examining for sufficient data to avoid type I (false-positive) and type II (false-negative) errors. Trial sequential analysis was performed using TSA software (version 0.9.5.9 Beta; Copenhagen Trial Unit, Copenhagen, Denmark). The Lan-DeMets approach was used for construction of the O'Brien-Fleming monitoring boundaries and the optimal information size, which was set to an alpha of 0.05 with a two-sided beta of 0.80 and relative risk reduction of $20 \%$.

\section{Sensitivity analysis}

Sensitivity analyses were conducted on the primary outcomes to test the robustness of the results by the following methods: changing to a fixed-effect model, changing to use of the Sidik-Jonkman method for random effects, shifting to the Biggerstaff-Tweedie method, excluding any estimated values, excluding crossover studies, and excluding studies with an early termination and/or high risk of bias.

We used Review Manager Software (Version 5.3, The Cochrane Collaboration, Copenhagen, Denmark) and TSA software (version 0.9.5.9 Beta, Copenhagen Trial Unit, Copenhagen, Denmark) to conduct the statistical analysis. The Grading of Recommendations Assessment, Development, and Evaluation (GRADE) Guideline Development Tool (GRADEpro; McMaster University 2014, Hamilton, Canada) was used to evaluate the quality of evidence for each outcome [24]. The quality of evidence was stratified into four grades: high, moderate, low, or very low. $P<0.05$ was considered statistically significant.

\section{Role of the funding source}

This study was supported by the National Natural Science Foundation of China (NSFC81871585) and the Natural Science Foundation of Guangdong Province (2018A030313058). The study sponsors did not involve in study design, collection, data analysis and interpretation, writing of the report, or decision to submit the paper for publication.

\section{Results}

Our study identified 1305 relevant publications. After removing duplicate results and screening the titles and abstracts, 548 publications were rescreened for titles and abstracts. Thirty studies were obtained for full-context review, and 20 studies were excluded. The details of the excluded studies and reasons for their exclusion are shown in the Additional file 1: Table S1. Finally, we included 10 studies (7 randomized controlled studies, 3 crossover studies) [12, 13, 17-19, 22, 23, 25-27] with a total of 1708 patients (median, 130 patients; range, 28527 patients; ECMO group, 856 patients; MV group, 852 patients) in this meta-analysis. The selection process of the eligible studies is shown in Fig. 1. Of the ten included studies, 66.5\% (range 47.6-85.7\%; IQR 56.5\%74.1\%) were men and $33.4 \%$ (range 14.3-52.4\%; IQR $25.9-43.5 \%$ ) were women. The durations and flow rates of HFNC in each study are shown in Table 1. The included study characteristics and baseline patient characteristics are shown in Tables 1 and 2.

Eight of the ten included studies were considered to be at low risk of bias as evaluated by the Cochrane risk of bias tool and modified Jadad score (Additional file 1: Figure S1 and S2, Table S2).

\section{Postextubation respiratory failure}

The data on postextubation respiratory failure were available from five studies. When these data were pooled together, the HFNC group showed a significant reduction of postextubation respiratory failure compared with that of the COT group (RR, 0.61; 95\% CI, 0.41, 0.92; $z=$ 2.38; $P=0.02$; Fig. 2). There was moderate heterogeneity among the studies $\left(\mathrm{chi}^{2}=7.82, \mathrm{df}=4, P=0.10, I^{2}=49 \%\right)$ which might be due to a heterogeneous population of patients among the included studies (Table 1) and various treatment measures after extubation. Subgroup analyses demonstrated no significant interactions with HFNC duration (HFNC $\geq 24 \mathrm{~h}$ RR, 0.52 [95\% CI, 0.330.84 ] vs. $\mathrm{HFNC}<24 \mathrm{~h}$ RR, 0.88 [95\% CI, $0.58-1.34$ ], $P_{\text {in- }}$ teraction $=0.10)$, HFNC flow $($ HFNC $\geq 40 \mathrm{~L} / \mathrm{min} \mathrm{RR}, 0.59$ [95\% CI, 0.34-1.05] vs. HFNC< 40 L/min RR, 0.58 [95\% CI, 0.35-0.95], $\left.P_{\text {interaction }}=0.94\right)$, severity of patients 


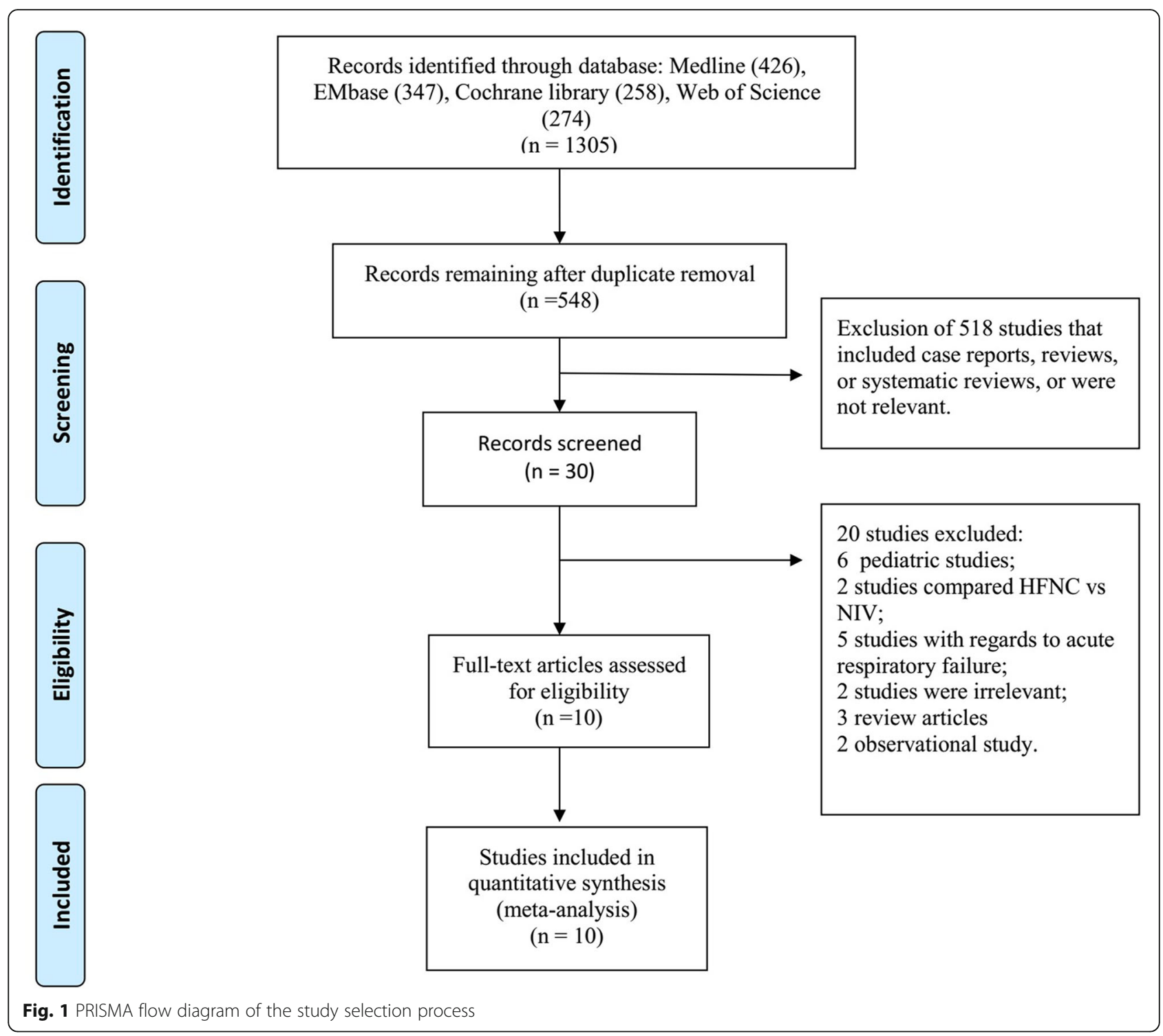

(severe subgroup RR, 0.42 [95\% CI, 0.12-1.52] vs. non severe subgroup RR, 0.72 [95\% CI, 0.53-0.99], $P_{\text {interaction }}$ $=0.42$ ), and hypercapnic or not (non-hypercapnic RR, 0.65 [95\% CI, 0.44-0.94] vs. mixed subgroup RR, 0.48 $\left.[95 \% \mathrm{CI}, 0.18-1.29], P_{\text {interaction }}=0.59\right) \quad($ Table 3, Additional file 1: Figure S3-S6).

The result was robust to multiple sensitivity analyses, including changing to a fixed-effect model, SJ effect model, or BT effect model, excluding any estimated values, excluding crossover studies, or excluding the high-risk bias study and/or early termination study (Additional file 1: Table S3). For primary outcome, trial sequential analysis confirmed that the required information size was not reached; however, the $Z$-curve had crossed O'Brian-Fleming monitoring boundaries, indicating that HFNC was beneficial than COT in postextubation respiratory failure (Additional file 1: Figure S7).
$\mathrm{PaO}_{2}$ was significantly higher with HFNC compared with COT (SMD, 0.30; 95\% CI, 0.04, 0.56; $z=2.23 ; P=$ 0.03 ; Fig. 3 ), and respiratory rate was significantly lower in HFNC group compared with COT group (SMD, 0.70; 95\% CI, $-1.16,-0.25 ; z=3.03 ; P=0.002$; Fig. 4). There were moderate to high heterogeneity which might be due to a heterogeneous population of patients among the included studies (Table 1). Sensitivity analyses did not change the overall findings (Additional file 1: Table S3). Subgroup analyses demonstrated there were significant interactions with regard to study type (RCT study SMD, - 1.12 [95\% CI, - 1.45, - 0.79] vs. Crossover study, -0.35 [95\% CI, $\left.-0.75,0.04], P_{\text {interaction }}=0.004\right)$, HFNC duration (HFNC $\geq 24 \mathrm{~h}$ SMD, - 1.12 [95\% CI, - 1.45, $0.79]$ vs. HFNC < $24 \mathrm{~h}$ SMD, -0.35 [95\% CI, -0.75 , $\left.0.04], P_{\text {interaction }}=0.004\right)$, hypercapnic or not (non-hypercapnic SMD, $-0.16[95 \% \mathrm{CI},-0.59,0.26]$ vs. mixed 


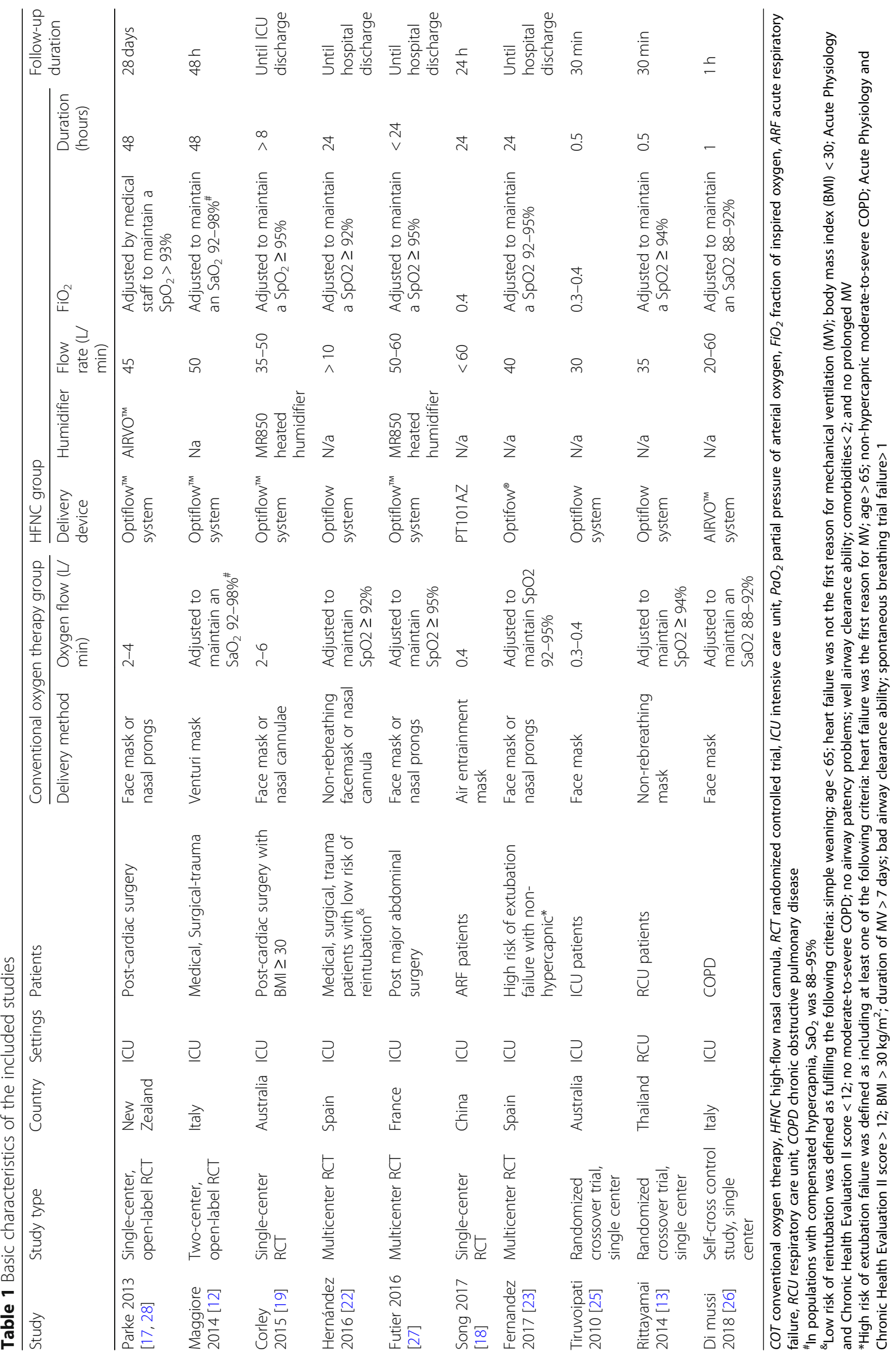


Table 2 Characteristics and demographic parameters of patients in the included studies

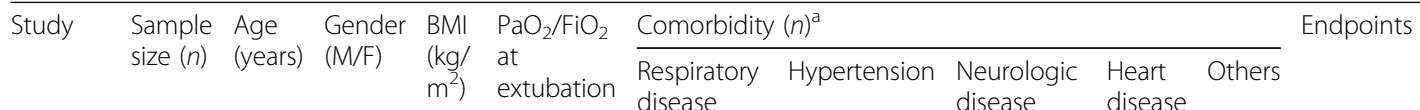

\begin{tabular}{|c|c|c|c|c|c|c|}
\hline \multicolumn{7}{|c|}{ Parke $2013[17,28]$} \\
\hline $\begin{array}{l}\text { HFNC } \\
\text { group }\end{array}$ & 169 & $\begin{array}{l}65 \\
(19- \\
88)^{*}\end{array}$ & $129 / 40$ & $\begin{array}{l}28.4 \\
\pm \\
5.3\end{array}$ & N/a & $\mathrm{N} / \mathrm{a}$ \\
\hline $\begin{array}{l}\text { COT } \\
\text { group }\end{array}$ & 171 & $\begin{array}{l}66 \\
(21- \\
87)^{*}\end{array}$ & $129 / 42$ & $\begin{array}{l}29.2 \\
\pm \\
5.5\end{array}$ & N/a & $\mathrm{N} / \mathrm{a}$ \\
\hline \multicolumn{7}{|c|}{ Maggiore 2014 [12] } \\
\hline $\begin{array}{l}\text { HFNC } \\
\text { group }\end{array}$ & 53 & $\begin{array}{l}65 \pm \\
18\end{array}$ & $33 / 20$ & N/a & $\begin{array}{l}239.4 \pm \\
42.4\end{array}$ & 24 \\
\hline $\begin{array}{l}\text { COT } \\
\text { group }\end{array}$ & 52 & $\begin{array}{l}64 \pm \\
17\end{array}$ & $35 / 17$ & N/a & $\begin{array}{l}241.7 \pm \\
51.1\end{array}$ & 24 \\
\hline
\end{tabular}
disease disease

Corley 2015 [19]

$\begin{array}{lllllll}\text { HFNC } & 81 & 63 \pm & 58 / 23 & 36 & \text { N/a } & 26 \\ \text { group } & & 11.4 & & \pm & & \\ & & & & 5.2 & & \\ \text { COT } & 74 & 65 \pm & 56 / 18 & 35 & \text { N/a } & 20 \\ \text { group } & 11.1 & & \pm & & \\ & & & & 4.3 & & \end{array}$

Hernández 2016 [22]

\begin{tabular}{|c|c|c|c|c|c|}
\hline $\begin{array}{l}\text { HFNC } \\
\text { group }\end{array}$ & 264 & $\begin{array}{l}51 \pm \\
13.1\end{array}$ & $\begin{array}{l}164 / \\
100\end{array}$ & $<30$ & $227 \pm 25$ \\
\hline $\begin{array}{l}\text { COT } \\
\text { group }\end{array}$ & 263 & $\begin{array}{l}51.8 \\
\pm 12.2\end{array}$ & $\begin{array}{l}153 / \\
110\end{array}$ & $<30$ & $237 \pm 34$ \\
\hline
\end{tabular}

$\begin{array}{lll}\text { N/a } & 6 & 23 \\ \text { N/a } & 5 & 23\end{array}$

Primary outcome: oxygenation. Secondary outcomes: oxygen desaturation, device displacement, requiring ventilator support", reintubation, discomfort score.

Secondary outcomes: Atelectasis score, length of ICU and hospital stay, 28-day mortality, oxygentory support, espirometry, of respirascore.

Futier 2016 [27]

\begin{tabular}{|c|c|c|c|c|c|c|c|c|c|c|c|}
\hline $\begin{array}{l}\text { HFNC } \\
\text { group }\end{array}$ & 108 & $\begin{array}{l}62 \pm \\
12\end{array}$ & $61 / 47$ & $\begin{array}{l}25 \\
\pm 4\end{array}$ & N/a & 10 & 34 & N/a & N/a & 76 & $\begin{array}{l}\text { Primary outcome: rate of } \\
\text { hypoxemia } 1 \mathrm{~h} \text { after extubation. }\end{array}$ \\
\hline $\begin{array}{l}\text { COT } \\
\text { group }\end{array}$ & 112 & $\begin{array}{l}61 \pm \\
13\end{array}$ & $64 / 48$ & $\begin{array}{l}25 \\
\pm 4\end{array}$ & N/a & 8 & 35 & $\mathrm{~N} / \mathrm{a}$ & N/a & 67 & $\begin{array}{l}\text { Secondary outcomes: pulmonary } \\
\text { complications, length of ICU and } \\
\text { hospital stay, in-hospital mortality }\end{array}$ \\
\hline
\end{tabular}

Song 2017 [18]

$\begin{array}{lllllll}\text { HFNC } & 30 & 66 \pm & 16 / 14 & \text { N/a } & 207 \pm 27.5 & 19 \\ \text { group } & & 14 & & & & \\ \text { COT } & 30 & 71 \pm & 18 / 12 & \text { N/a } & 204 \pm 29 & 19 \\ \text { group } & 13 & & & & \end{array}$

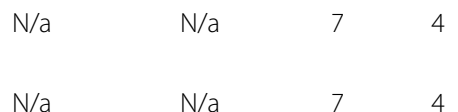

Primary outcome: therapy success rate. Secondary outcomes: RR, HR, oxygenation indices, MAP.

Fernandez 2017 [23]

\begin{tabular}{|c|c|c|c|c|c|}
\hline $\begin{array}{l}\text { HFNC } \\
\text { group }\end{array}$ & 78 & $\begin{array}{l}67.3 \\
\pm 12\end{array}$ & $46 / 32$ & N/a & N/a \\
\hline $\begin{array}{l}\text { COT } \\
\text { group }\end{array}$ & 77 & $\begin{array}{l}69.7 \\
\pm 13\end{array}$ & $55 / 22$ & N/a & N/a \\
\hline
\end{tabular}

$\begin{array}{ccccl}\text { N/a } & \text { N/a } & 9 & \text { N/a } & \begin{array}{l}\text { Primary outcome: respiratory } \\ \text { failure within } 3 \text { days. Secondary } \\ \text { outcomes: length of ICU and } \\ \text { hospital stay, reintubation, } \\ \text { mortality. }\end{array}\end{array}$

Tiruvoipati 2010 [25]

\begin{tabular}{|c|c|c|c|c|c|c|c|c|c|c|c|}
\hline $\begin{array}{l}\text { HFNC } \\
\text { group }\end{array}$ & 42 & $\begin{array}{l}65.22 \\
\pm 17.6\end{array}$ & $20 / 22$ & $\mathrm{~N} / \mathrm{a}$ & $>175$ & N/a & N/a & $\mathrm{N} / \mathrm{a}$ & N/a & N/a & \multirow{2}{*}{$\begin{array}{l}\text { Primary outcome: efficacy of } \\
\text { oxygenation. Secondary } \\
\text { outcomes: HR, RR, blood pressure, } \\
\text { comfort score, tolerance score. }\end{array}$} \\
\hline $\begin{array}{l}\text { COT } \\
\text { group }\end{array}$ & 42 & $\begin{array}{l}65.22 \\
\pm 17.6\end{array}$ & $20 / 22$ & $\mathrm{~N} / \mathrm{a}$ & $>175$ & N/a & N/a & $\mathrm{N} / \mathrm{a}$ & $\mathrm{N} / \mathrm{a}$ & N/a & \\
\hline \multicolumn{12}{|c|}{ tttayamai 2014 [13] } \\
\hline HFNC & 17 & 66.8 & $10 / 7$ & N/a & $\geq 150$ & 9 & 8 & N/a & 8 & N/a & Primary outcome: dyspnea score. \\
\hline
\end{tabular}


Table 2 Characteristics and demographic parameters of patients in the included studies (Continued)

\begin{tabular}{|c|c|c|c|c|c|c|c|c|c|c|c|}
\hline \multirow[t]{2}{*}{ Study } & \multirow{2}{*}{$\begin{array}{l}\text { Sample } \\
\text { size }(n)\end{array}$} & \multirow{2}{*}{$\begin{array}{l}\text { Age } \\
\text { (years) }\end{array}$} & \multirow{2}{*}{$\begin{array}{l}\text { Gender } \\
(\mathrm{M} / \mathrm{F})\end{array}$} & \multirow{2}{*}{$\begin{array}{l}\mathrm{BMl} \\
(\mathrm{kg} / \\
\left.\mathrm{m}^{2}\right)\end{array}$} & \multirow{2}{*}{$\begin{array}{l}\mathrm{PaO}_{2} / \mathrm{FiO}_{2} \\
\text { at } \\
\text { extubation }\end{array}$} & \multicolumn{5}{|c|}{ Comorbidity $(n)^{\mathrm{a}}$} & \multirow[t]{2}{*}{ Endpoints } \\
\hline & & & & & & $\begin{array}{l}\text { Respiratory } \\
\text { disease }\end{array}$ & Hypertension & $\begin{array}{l}\text { Neurologic } \\
\text { disease }\end{array}$ & $\begin{array}{l}\text { Heart } \\
\text { disease }\end{array}$ & Others & \\
\hline group & & \pm 13.8 & & & & & & & & & \multirow{2}{*}{$\begin{array}{l}\text { Secondary outcomes: HR, RR, } \\
\text { MAP, comfort score. }\end{array}$} \\
\hline $\begin{array}{l}\text { COT } \\
\text { group }\end{array}$ & 17 & $\begin{array}{l}66.8 \\
\pm 13.8\end{array}$ & $10 / 7$ & N/a & $\geq 150$ & 9 & 8 & N/a & 8 & $\mathrm{~N} / \mathrm{a}$ & \\
\hline \multicolumn{12}{|c|}{ Di mussi 2018 [26] } \\
\hline $\begin{array}{l}\text { HFNC } \\
\text { group }\end{array}$ & 14 & $\begin{array}{l}71.5 \\
\pm 9\end{array}$ & $12 / 2$ & N/a & $>150$ & N/a & N/a & N/a & N/a & $\mathrm{N} / \mathrm{a}$ & \multirow{2}{*}{$\begin{array}{l}\text { Primary outcome: } \\
\text { neuroventilatory drive and work } \\
\text { of breathing. Secondary } \\
\text { outcomes: RR, oxygenation } \\
\text { indices. }\end{array}$} \\
\hline $\begin{array}{l}\text { COT } \\
\text { group }\end{array}$ & 14 & $\begin{array}{l}71.5 \\
\pm 9\end{array}$ & $12 / 2$ & N/a & $>150$ & $\mathrm{~N} / \mathrm{a}$ & $\mathrm{N} / \mathrm{a}$ & $\mathrm{N} / \mathrm{a}$ & N/a & $\mathrm{N} / \mathrm{a}$ & \\
\hline
\end{tabular}

Respiratory disease including pneumonia, chronic obstructive pulmonary disease, asthma, and other respiratory disease; heart disease including cardiogenic pulmonary edema, congestive heart failure, and cardiac arrest

$\mathrm{M}$ male, $\mathrm{F}$ female, $\mathrm{BMI}$ body mass index, $\mathrm{PaO}_{2}$ arterial partial pressure of oxygen, $\mathrm{FiO}_{2}$ fraction of the inspired oxygen, $\mathrm{HFNC}$ high-flow nasal cannula, $\mathrm{COT}$ conventional oxygen therapy, $B M I$ body-mass index, $\mathrm{SpO}_{2}$ pulse oxygen saturation, $R R$ respiratory rate, $A R R$ absolute risk reduction, $H R$ heart rate, $M A P$ mean arterial pressure

Plus-minus values are the means \pm SDs

a Patients can have more than 1 comorbidity

*Values are median and interquartile range

\#Requiring ventilator support including any form of ventilator support, e.g., noninvasive ventilation or mechanical ventilation

subgroup SMD, -1.07 [95\% CI, $-1.37,-0.77], P_{\text {interac- }}$ tion $=0.007)$ in respiratory rate (Table 3$)$.

There were no significant differences in reintubation rate, length of ICU and hospital stay, comfort score, $\mathrm{PaCO}_{2}$, and mortality in ICU and hospital between HFNC and COT group (Additional file 1: Figure S8-S14)

\section{Severe adverse events}

Among the included studies, no severe adverse effects were reported in both groups.

Visual inspection of the funnel plot did not show any publication bias (Additional file 1: Figure S15-S24). Summary of findings with GRADE system are shown in Table 4.

\section{Discussion}

This systematic review and meta-analysis, including 1708 planned patients (HFNC group: 856 patients; COT group: 852 patients), demonstrated that compared with
COT, HFNC might significantly reduce postextubation respiratory failure $(\mathrm{RR}, 0.61 ; 95 \% \mathrm{CI}, 0.41,0.92 ; z=2.38$; $P=0.02)$ and respiratory rates (SMD, $-0.70 ; 95 \% \mathrm{CI},-$ $1.16,-0.25 ; z=3.02 ; P=0.002)$ and increase $\mathrm{PaO}_{2}$ (SMD, 0.30; 95\% CI, 0.04, 0.56; $z=2.23 ; P=0.03$ ). There were no significant differences in reintubation rate, length of ICU and hospital stay, comfort score, $\mathrm{PaCO}_{2}$, mortality in ICU and hospital, and severe adverse events between the HFNC and COT group.

The present systematic review and meta-analysis demonstrated a biologically plausible association between HFNC therapy and decreased postextubation respiratory failure in planned extubation patients. Previous animal and human mechanistic studies have demonstrated that HFNC enables to deliver more adequate inspiratory flow, flush the nasopharyngeal dead space, and deliver warm and humidified gas, thereby generating a positive airway pressure, ameliorating oxygenation and dyspnea, reducing the respiratory rate and work of breathing, and improving comfort [29-32].

\begin{tabular}{|c|c|c|c|c|c|c|c|c|c|c|}
\hline \multirow[b]{2}{*}{ Studv or Subgroup } & \multicolumn{2}{|c|}{ HFNC group } & \multicolumn{2}{|c|}{ COT group } & \multirow{2}{*}{ Weight } & \multirow{2}{*}{$\begin{array}{c}\text { Risk Ratio } \\
\text { M-H, Random, } 95 \% \mathrm{Cl}\end{array}$} & \multirow{2}{*}{ Year } & \multirow{2}{*}{\multicolumn{3}{|c|}{$\begin{array}{c}\text { Risk Ratio } \\
\text { M-H, Random, } 95 \% \mathrm{Cl}\end{array}$}} \\
\hline & Events & Total & Events & Total & & & & & & \\
\hline Maggiore 2014 & 4 & 53 & 19 & 52 & $11.8 \%$ & $0.21[0.08,0.57]$ & 2014 & & & \\
\hline Hernández 2016 & 22 & 264 & 38 & 263 & $26.5 \%$ & $0.58[0.35,0.95]$ & 2016 & & & \\
\hline Futier 2016 & 29 & 108 & 34 & 112 & $30.0 \%$ & $0.88[0.58,1.34]$ & 2016 & & - & \\
\hline Fernandez 2017 & 16 & 78 & 21 & 77 & $23.6 \%$ & $0.75[0.43,1.33]$ & 2017 & $\rightarrow$ & + & \\
\hline Song 2017 & 3 & 30 & 6 & 30 & $8.1 \%$ & $0.50[0.14,1.82]$ & 2017 & & & \\
\hline Total $(95 \% \mathrm{Cl})$ & & 533 & & 534 & $100.0 \%$ & $0.61[0.41,0.92]$ & & & & \\
\hline Total events & 74 & & 118 & & & & & & & \\
\hline $\begin{array}{l}\text { Heterogeneity: Tau }{ }^{2} \\
\text { Test for overall effect }\end{array}$ & $\begin{array}{l}0.10 ; \mathrm{Chi}^{\mathrm{z}} \\
\mathrm{Z}=2.38\end{array}$ & $\begin{array}{l}=7.82 \\
P=0.02\end{array}$ & df $d f=4(P$ & $=0.10)$ & ) $I^{2}=49 \%$ & & 0.01 & $\begin{array}{c}0.1 \\
\text { Favours HFNC }\end{array}$ & ${ }^{1} \begin{array}{c}10 \\
\text { Favours COT }\end{array}$ & 100 \\
\hline
\end{tabular}


Table 3 Subgroup analysis for outcomes (displayed with RR or SMD)

\begin{tabular}{|c|c|c|c|c|}
\hline Outcomes & $\begin{array}{l}\text { Postextubation respiratory failure } \\
\text { (RR) }\end{array}$ & $\begin{array}{l}\text { Reintubation } \\
\text { (RR) }\end{array}$ & $\begin{array}{l}\text { Respiratory rate } \\
\text { (SMD) }\end{array}$ & $\begin{array}{l}\mathrm{PaO}_{2} \\
(\mathrm{SMD})\end{array}$ \\
\hline \multicolumn{5}{|l|}{ Subgroup analyses } \\
\hline \multicolumn{5}{|l|}{ Study type } \\
\hline RCT study & $0.61(0.41,0.92)$ & $0.58(0.30,1.11)$ & $-1.12(-1.45,-0.79)$ & $0.39(-0.02,0.79)$ \\
\hline Crossover study & $\mathrm{Na}$ & $\mathrm{Na}$ & $-0.35(-0.75,0.04)$ & $0.14(-0.23,0.51)$ \\
\hline Interaction & $\mathrm{Na}$ & $\mathrm{Na}$ & $P=0.004$ & $P=0.38$ \\
\hline \multicolumn{5}{|l|}{ Severity of patients } \\
\hline Severe populations & $0.42(0.12,1.52)$ & $0.39(0.13,1.19)$ & $-0.56(-1.29,0.18)$ & $0.31(0.05,0.58)$ \\
\hline Non severe population & $0.72(0.53,0.99)$ & $0.81(0.27,2.45)$ & $-0.93(-1.36,-0.50)$ & $0.35(-0.29,1.00)$ \\
\hline Interaction & 0.42 & 0.36 & 0.39 & 0.92 \\
\hline \multicolumn{5}{|l|}{ HFNC flow } \\
\hline$\geq 40 \mathrm{~L} / \mathrm{min}$ & $0.59(0.34,1.05)$ & $0.72(0.29,1.83)$ & $-1.14(-1.47,-0.81)$ & $0.39(-0.02,0.79)$ \\
\hline$<40 \mathrm{~L} / \mathrm{min}$ & $0.58(0.35,0.95)$ & $0.39(0.21,0.72)$ & $-0.44(-1.09,0.21)$ & $0.10(-0.33,0.52)$ \\
\hline Interaction & 0.94 & 0.28 & 0.06 & 0.33 \\
\hline \multicolumn{5}{|l|}{ Non-hypercapnic or not } \\
\hline Non-hypercapnic & $0.65(0.44,0.94)$ & $0.52(0.29,0.93)$ & $-0.16(-0.59,0.26)$ & $0.10(-0.33,0.52)$ \\
\hline Mixed $^{\mathrm{a}}$ & $0.48(0.18,1.29)$ & $0.45(0.12,1.77)$ & $-1.07(-1.37,-0.77)$ & $0.39(-0.02,0.79)$ \\
\hline Interaction & 0.59 & 0.86 & 0.0007 & 0.33 \\
\hline \multicolumn{5}{|l|}{ HFNC duration } \\
\hline$\geq 24 \mathrm{~h}$ & $0.52(0.33,0.84)$ & $0.48(0.26,0.89)$ & $-1.12(-1.45,-0.79)$ & $0.58(0.27,0.90)$ \\
\hline$<24 \mathrm{~h}$ & $0.88(0.58,1.34)$ & $0.88(0.11,7.33)$ & $-0.35(-0.75,0.04)$ & $0.09(-0.13,0.30)$ \\
\hline Interaction & $P=0.10$ & $P=0.59$ & $P=0.004$ & $P=0.01$ \\
\hline \multicolumn{5}{|l|}{ Post cardiac surgery or not } \\
\hline Post cardiac surgery & $\mathrm{Na}$ & $0.96(0.04,24.84)$ & $\mathrm{Na}$ & $\mathrm{Na}$ \\
\hline Other patients & $0.62(0.42,0.92)$ & $0.55(0.28,1.08)$ & $-0.70(-1.16,-0.25)$ & $0.30(0.04,0.56)$ \\
\hline Interaction & $\mathrm{Na}$ & $P=0.74$ & $\mathrm{Na}$ & $\mathrm{Na}$ \\
\hline
\end{tabular}

RRs and 95\% confidence intervals (Cls) were calculated for the binary data, and the standardized mean differences (SMDs) and 95\% Cls were calculated for the continuous data variables

$R R$ risk ratio, $S M D$ standardized mean difference, $R C T$ randomized controlled trial, $H F N C$ high-flow nasal cannula, $P a O_{2}$ partial pressure of arterial oxygen

${ }^{a}$ Means studies included patients with hypoxemic or/and hypercapnic respiratory failure

Our study showed that HFNC might significantly reduce postextubation respiratory failure in patients after planned extubation. The result was consistent with multiple subgroup analyses, sensitivity analyses, and trial sequential analysis. However, as there was moderate heterogeneity $\left(\mathrm{chi}^{2}=7.82, \quad \mathrm{df}=4, \quad P=0.10, \quad I^{2}=49 \%\right)$ among the included studies, which might have been due to the heterogeneous population of patients and various treatment measures after extubation, a decisive conclusion should be made cautiously. Further large-scale, multicenter studies are needed to confirm our results.

A previous study showed that in patients with acute hypoxemic respiratory failure, an increasing HFNC flow rate $(30,45$, and $60 \mathrm{~L} / \mathrm{min}$ ) progressively decreased inspiratory effort and improved lung aeration, dynamic compliance, and oxygenation [33]. In a study by Parke

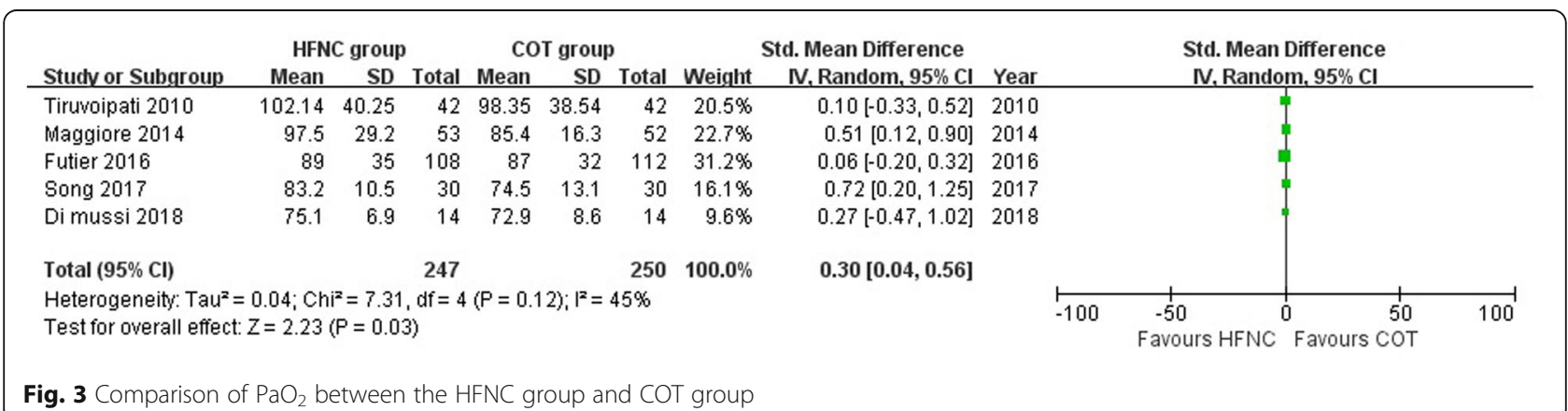




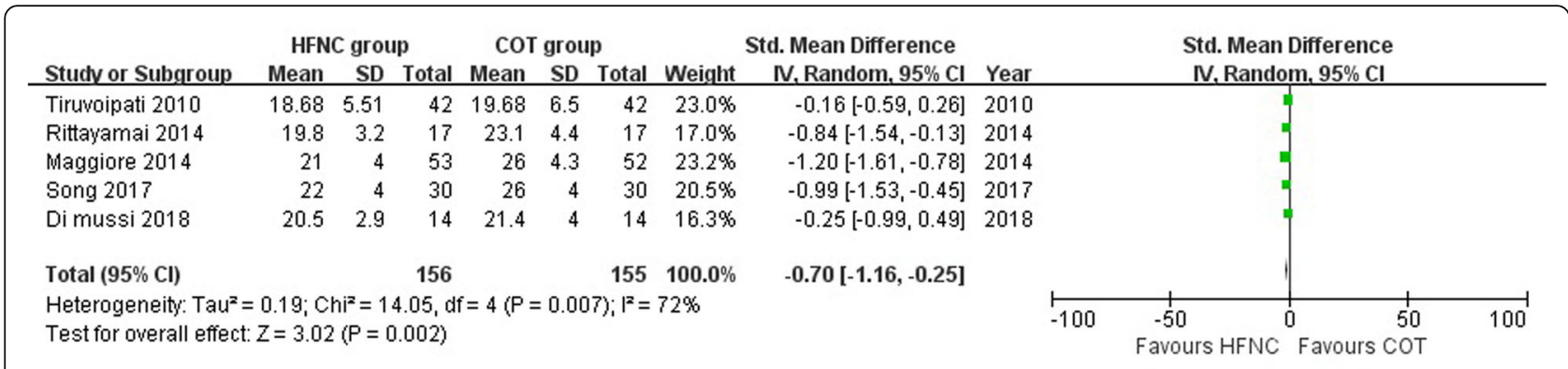

Fig. 4 Comparison of respiratory rates between the HFNC group and COT group=

and coworkers, patients' nasopharyngeal pressures were measured when HFNC was used with gas flows of 30, 40 , and $50 \mathrm{~L} / \mathrm{min}$ [28]. Researchers demonstrated that the mean nasopharyngeal pressures were $1.5 \pm 0.6,2.2 \pm$ 0.8 , and $3.1 \pm 1.2 \mathrm{mmHg}$ at 30,40 , and $50 \mathrm{~L} / \mathrm{min}$ during HFNC therapy, respectively. They showed that the level of PEEP as a benefit of HFNC therapy was flow-dependent. The various starting flows may have led to different levels of PEEP and could have influenced the results. Thus, we performed a subgroup analysis according to the flow rate of HFNC $(\geq 40 \mathrm{~L} / \mathrm{min},<40 \mathrm{~L} / \mathrm{min})$, and we did not find significant interactions between subgroups with regard to postextubation respiratory failure $\left(P_{\text {interaction }}=0.94\right)$, reintubation $\left(P_{\text {interaction }}=0.28\right)$, respiratory rate $\left(P_{\text {interaction }}=0.06\right)$, and $\mathrm{PaO}_{2}\left(P_{\text {interaction }}=\right.$
0.33). These results may be due to the benefits of HFNC being produced not only by a high-flow rate but also through the effect of heating and humidification, reducing the work of breathing and being more comfortable for patients [34, 35].

Subgroup analysis with regard to HFNC duration showed a reduction of postextubation respiratory failure in studies that used this therapy for $\geq 24 \mathrm{~h}$ (RR, 0.53; 95\% CI, 0.34, 0.84; $z=2.73 ; P=0.006$ ) and found no efficacy in those that used HFNC < 24h (RR, 0.88; 95\% CI, $0.58,1.34 ; z=0.57 ; P=0.57$ ). Our previous study also showed that HFNC therapy might decrease the rate of escalation of respiratory support and the intubation rate when ARF patients were treated with HFNC for $\geq 24 \mathrm{~h}$ [36]. However, no significant interaction $\left(P_{\text {interaction }}=\right.$

Table 4 Summary of findings

\begin{tabular}{|c|c|c|c|c|c|c|}
\hline \multirow[t]{2}{*}{ Outcomes } & \multicolumn{2}{|c|}{ Anticipated absolute effects ${ }^{*}(95 \% \mathrm{Cl})$} & \multirow{2}{*}{$\begin{array}{l}\text { Relative } \\
\text { effect } \\
(95 \% \mathrm{Cl})\end{array}$} & \multirow{2}{*}{$\begin{array}{l}\text { No. of } \\
\text { participants } \\
\text { (studies) }\end{array}$} & \multirow{2}{*}{$\begin{array}{l}\text { Certainty } \\
\text { of the } \\
\text { evidence } \\
\text { (GRADE) }\end{array}$} & \multirow[t]{2}{*}{ Comments } \\
\hline & Risk with COT & Risk with HFNC & & & & \\
\hline $\begin{array}{l}\text { Postextubation } \\
\text { respiratory } \\
\text { failure }\end{array}$ & 219 per 1000 & $\begin{array}{l}136 \text { per } 1000 \\
\text { (92 to 202) }\end{array}$ & $\begin{array}{l}\text { RR } 0.62 \\
(0.42 \text { to } \\
0.92)\end{array}$ & $\begin{array}{l}1067 \\
\text { (5 RCTs) }\end{array}$ & $\begin{array}{l}\oplus \oplus \oplus \oplus \\
\text { High }\end{array}$ & \\
\hline $\mathrm{PaO}_{2}(\mathrm{mmHg})$ & $\begin{array}{l}\text { The mean } \mathrm{paO}_{2} \\
\text { was } 83.63 \\
\mathrm{mmHg}\end{array}$ & $\begin{array}{l}\text { The mean paO2 in the } \\
\text { intervention group was } 89.39 \\
\mathrm{mmHg}(75.91 \text { to } 102.86 \mathrm{mmHg})\end{array}$ & - & $\begin{array}{l}497 \\
(5 \mathrm{RCTs})\end{array}$ & $\begin{array}{l}\oplus \oplus \oplus \oplus \\
\text { High }\end{array}$ & \\
\hline $\begin{array}{l}\text { Respiratory } \\
\text { rates } \\
\text { (breaths per } \\
\text { minute) }\end{array}$ & $\begin{array}{l}\text { The mean } \\
\text { respiratory rates } \\
\text { was } 23.24 \\
\text { breaths per } \\
\text { minute }\end{array}$ & $\begin{array}{l}\text { The mean respiratory rates in the } \\
\text { intervention group was } 20.4 \\
\text { breaths per minute ( } 18.84 \text { to } \\
21.95 \text { breaths per minute) }\end{array}$ & - & $\begin{array}{l}311 \\
(5 \mathrm{RCTS})\end{array}$ & $\begin{array}{l}\oplus \oplus \oplus \oplus \\
\text { High }\end{array}$ & $\begin{array}{l}\text { Respiratory rates obtained from the study } \\
\text { by Maggiore and colleague was reported } \\
\text { with cartograms, and we extracted data } \\
\text { with Digitizelt software (Braunschweig, } \\
\text { Germany). }\end{array}$ \\
\hline Reintubation & 82 per 1000 & $\begin{array}{l}48 \text { per } 1000 \\
(25 \text { to } 91)\end{array}$ & $\begin{array}{l}\text { RR } 0.58 \\
(0.30 \text { to } \\
1.11)\end{array}$ & $\begin{array}{l}1562 \\
\text { (7 RCTs) }\end{array}$ & $\begin{array}{l}\oplus \oplus \oplus \oplus \\
\text { High }\end{array}$ & \\
\hline
\end{tabular}

Patient or population: patients after planned extubation

Setting:

Intervention: HFNC

Comparison: COT

GRADE Working Group grades of evidence:

High certainty: We are very confident that the true effect lies close to that of the estimate of the effect

Moderate certainty: We are moderately confident in the effect estimate-the true effect is likely to be close to the estimate of the effect, but there is a possibility that it is substantially different

Low certainty: Our confidence in the effect estimate is limited-the true effect may be substantially different from the estimate of the effect

Very low certainty: We have very little confidence in the effect estimate-the true effect is likely to be substantially different from the estimate of effect

*The risk in the intervention group (and its $95 \%$ confidence interval) is based on the assumed risk in the comparison group and the relative effect of the intervention (and its $95 \% \mathrm{Cl}$ )

$C l$ confidence interval, $R R$ risk ratio 
0.11) was found between subgroups in the present meta-analysis. This may be due to only one study involved in the subgroup that used HFNC < 24 h [27]. Further studies comparing the effect of duration for HFNC treatment in patients after planned extubation are needed.

Patients who presented with hypoxemic or hypercapnic respiratory failure after planed extubation might lead to different results. Therefore, we performed a subgroup analysis by stratified studies into a nonhypercapnic subgroup and mixed subgroup (hypoxemic or/and hypercapnic). In the study by Parke and colleagues, the baseline $\mathrm{PaCO}_{2}$ levels were not reported; thus, it was difficult to know whether patients with hypercapnic respiratory failure were studied [17]. In the studies by Hernández and colleagues and by Fernandez and coworkers, nonhypercapnic patients were studied [22, 23]. In the 7 other studies, mixed patients were included [12, 13, 18, 19, 25-27]. Subgroup analysis found that there were no differences in postextubation respiratory failure $\left(P_{\text {interaction }}=0.59\right)$, reintubation $\left(P_{\text {interaction }}=0.86\right)$, and $\mathrm{PaO}_{2}\left(P_{\text {interaction }}=0.33\right)$ between subgroups. And there was a significant difference in respiratory rate $\left(P_{\text {interaction }}\right.$ $=0.0007)$; however, this result needs to be interpreted with great caution because only one study was included in the nonhypercapnic subgroup [25].

Patients' severity might influence the effect of HFNC. Therefore, we performed subgroup analyses according to the severity of patients among included studies. All of the included studies reported severity scores using different severity evaluation methods. Four of the included studies reported Acute Physiology And Chronic Health Evaluation (APACHE) II scores. In the study by Song and coworkers, the APACHE II scores in the COT group and HFNC group were $12.36 \pm 3.29$ and $12.87 \pm 3.0$, respectively [18]. In the study by Corley and colleagues, the APACHE II scores in the COT group and HFNC group were $15 \pm 3.9$ and $15 \pm 3.6$, respectively [19]. In the study by Hernández and colleagues, the APACHE II scores in the COT group and HFNC group were 13 (717) and 14 (9-16), respectively [22]. In the study by Fernandez and coworkers, the APACHE II scores in the COT group and HFNC group were $21 \pm 8.2$ and $21 \pm 8.8$, respectively [23]. In the study by Tiruvoipati and coworkers, the APACHE III scores were reported, and the scores in the protocol A group and protocol B group were $70.55 \pm 27.39$ and $72.95 \pm 23.22$, respectively [25]. Three of the included studies reported the Simplified Acute Physiology Score (SAPS) II. In the study by Maggiore and colleagues, the SAPS II scores in the COT group and HFNC group were $44 \pm 16$ and $43 \pm 14$, respectively [12]. In the crossover study by Rittayamai and coworkers, the SAPS II score was $30.9 \pm 4.4$ [13]. In the crossover study by Di mussi and colleagues, the SAPS II score was $39.6 \pm 13.2$, and the Sequential Organ Failure Assessment (SOFA) score was $5.6 \pm 2.5$ [26]. In the study by Parke and coworkers, the EuroSCORE was reported, and the scores in the COT group and HFNC group were $5.3 \pm 2.8$ and $5.1 \pm 2.8$, respectively [17]. In the study by Futier and colleagues, the preoperative risk score was reported; few patients in both groups (15\% patients in the COT group and $17 \%$ patients in the HFNC group) were at high-risk levels, and the main patients in both groups were at moderate levels [27]. According to the severity scores of populations, we stratified the included studies into a severe subgroup (APACHE II $\geq 15$, SAPS II $\geq 38$, SOFA $\geq 2$ ) and non-severe subgroup (APACHE II $<15$, SAPS II <38, SOFA <2) $[37,38]$. However, we found no interactions between subgroups with regard to postextubation respiratory failure $\left(P_{\text {interaction }}=0.42\right)$, reintubation $\left(P_{\text {interaction }}=0.36\right)$, respiratory rate $\left(P_{\text {interaction }}=0.39\right)$, and $\mathrm{PaO}_{2}\left(P_{\text {interaction }}=0.92\right)$, which meant that the severity of patients would not influence the effect of HFNC with regard to postextubation respiratory failure, reintubation, respiratory rate, and $\mathrm{PaO}_{2}$.

Although a lower postextubation respiratory failure would be expected to decrease reintubation rate and shorten the length of ICU and hospital stays, no differences were found in this aspect in the present meta-analysis. This may be due to a heterogeneous population of patients included in our study and various clinical treatment measures applied when patients suffered postextubation failure. In three of the included studies, when patients in COT group need an escalation of respiratory support, HFNC therapy was applied which might make it difficult to interpret the results [17-19]. Hernández et al. speculated that this may be because the percentage of reintubated patients was too low to affect outcome variables in the entire group [22].

There are several limitations to our meta-analysis. First, this study involved a heterogeneous population of patients among the included studies (Table 1), which could affect our results. To address this problem, subgroup analyses and multiple sensitivity analysis were performed. And the subgroup results remained consistent with the overall findings. Multiple sensitivity analysis including changing effect models, excluding the high-risk bias study and/or early termination studies, did not change the overall results (Table 4). So we believed the results of our study were credible. Furthermore, the heterogeneous population of patients in our study enabled our results to have a general external validity in mixed populations of critically ill patients. Second, the duration of HFNC varied among the included studies (Table 1). Our previous study showed that HFNC therapy might decrease the rate of escalation of respiratory support and the intubation rate when ARF patients were treated with HFNC for $\geq 24 \mathrm{~h}$ [36]. However, a subgroup analysis of 
the present study did not find any interactions with regard to the duration of HFNC (Table 3). Further studies comparing the effect of duration in HFNC treatment in patients after planned extubation are needed. Third, among the included studies, $\mathrm{FiO}_{2}$ was titrated according to $\mathrm{SpO}_{2}$ or $\mathrm{SaO}_{2}$ (Table 1). We have reviewed all the studies included in this meta-analysis. Unexpectedly, except for the studies by Song and Tiruvoipati, the clear $\mathrm{FiO}_{2}$ values in these studies were not well reported. Subgroup analysis with regard to $\mathrm{FiO}_{2}$ was not performed. Finally, we include three crossover studies in the present study and crossover studies are limited by nature. Hence, we used the GRADE Guideline Development Tool to evaluate the quality of evidence which showed equal quality levels between crossover studies and randomized studies, showing that the results from the crossover studies should also be seriously considered.

\section{Conclusions}

Our meta-analysis demonstrated that compared with COT therapy, HFNC therapy may significantly reduce postextubation respiratory failure and respiratory rates, may increase $\mathrm{PaO}_{2}$, and may be safely administered in patients after planned extubation. Further large-scale, multicenter studies are needed to confirm our results.

\section{Additional file}

Additional file 1: Table S1. Studies Excluded after Full-text Review. Table S2. Quality of the included RCT studies. Table S3. Sensitivity analysis of the outcomes. Figure S1. Risk of bias graph. Figure S2. Risk of bias summary. Figure $\mathbf{S 3}$. Subgroup analysis of postextubation respiratory failure between the HFNC group and COT group according to HFNC duration. Figure S4. Subgroup analysis of postextubation respiratory failure between the HFNC group and COT group according to HFNC flow. Figure S5. Subgroup analysis of postextubation respiratory failure between the HFNC group and COT group according to severity of patients. Figure S6. Subgroup analysis of postextubation respiratory failure between the HFNC group and COT group according to hypercapnic or not. Figure S7. Trial sequential analysis. Figure S8. Comparison of reintubation between the two groups. Figure S9. Comparison of length of ICU stay between the two groups. Figure S10. Comparison of length of hospital stay between the two groups. Figure S11. Comparison of comfort score between the two groups. Figure S12. Comparison of $\mathrm{PaCO}_{2}$ between the two groups. Figure S13. Comparison of ICU mortality between the two groups. Figure S14. Comparison of hospital mortality between the two groups. Figure S15. Funnel plot of comparison for postextubation respiratory failure between the two group. Figure S16. Funnel plot of comparison for $\mathrm{PaO}_{2}$. Figure S17. Funnel plot of comparison for respiratory rates. Figure S18. Funnel plot of comparison for reintubation. Figure S19. Funnel plot of comparison for length of ICU stay. Figure S20. Funnel plot of comparison for length of hospital stay. Figure S21. Funnel plot of comparison for comfort score. Figure S22. Funnel plot of comparison for $\mathrm{PaCO}_{2}$. Figure S23. Funnel plot of comparison for ICU mortality. Figure S24. Funnel plot of comparison for hospital mortality. (DOC $613 \mathrm{~kb})$

\section{Abbreviations}

BMI: Body mass index; COT: Conventional oxygen therapy; $\mathrm{FiO}_{2}$ : Fraction of inspired oxygen; HFNC: High-flow nasal cannula; ICU: Intensive care unit;
NIV: Noninvasive ventilation; $\mathrm{PaO}_{2}$ : Partial pressure of oxygen in arterial blood; WMD: Weighted mean differences

\section{Acknowledgements}

We acknowledge all staff who helped perform this study and particularly acknowledge professor Qingshan Chen, who works in the Department of Medical Statistics in Jinan University, for providing advice regarding the data analysis

\section{Funding}

This study was supported by the National Natural Science Foundation of China (NSFC81871585) and the Natural Science Foundation of Guangdong Province (2018A030313058).

\section{Availability of data and materials}

All data generated or analyzed during this study are included in this published article.

\section{Authors' contributions}

All authors contributed to the study conception and design. HYY and RZ collected and helped extract the data. YFZ and JRW performed the analyses. YFZ and XLY conducted the literature review. All authors contributed to drafting the paper and approved the final manuscript.

\section{Ethics approval and consent to participate}

This study is a systematic review and meta-analysis, and ethics approval and consent to participate are not available.

\section{Consent for publication}

Not available.

\section{Competing interests}

The authors declare that they have no competing interests.

\section{Publisher's Note}

Springer Nature remains neutral with regard to jurisdictional claims in published maps and institutional affiliations.

\section{Author details}

'Department of Intensive Care Unit, Guangzhou Red Cross Hospital, Medical College, Jinan University, Tongfuzhong Road No. 396, Guangzhou 510220, Guangdong, China. ${ }^{2}$ Department of Cardiology, Guangzhou Red Cross Hospital, Medical College, Jinan University, Tongfuzhong Road No. 396, Guangzhou 510220, Guangdong, China.

Received: 6 March 2019 Accepted: 2 May 2019

Published online: 17 May 2019

References

1. Epstein SK. Weaning from ventilatory support. Curr Opin Crit Care. 2009;15: 36-43.

2. Hess DR. The role of noninvasive ventilation in the ventilator discontinuation process. Respir Care. 2012;57:1619-25.

3. Artime CA, Hagberg CA. Tracheal Extubation. Respir Care. 2014;59:991-1002.

4. Esteban A, Frutos VF, Muriel A, Ferguson ND, Peñuelas $O$, Abraira $V$, et al. Evolution of mortality over time in patients receiving mechanical ventilation. Am J Respir Crit Care Med. 2013;188:220-30.

5. Thille AW, Harrois A, Schortgen F, Brun-Buisson C, Brochard L. Outcomes of extubation failure in medical intensive care unit patients. Crit Care Med. 2011;39:2612-8

6. Rothaar RC, Epstein SK. Extubation failure: magnitude of the problem, impact on outcomes, and prevention. Curr Opin Crit Care. 2003;9:59-66.

7. Epstein SK, Ciubotaru RL, Wong JB. Effect of failed extubation on the outcome of mechanical ventilation. Chest. 1997:112:186-92.

8. Roca O, Riera J, Torres F, Masclans JR. High-flow oxygen therapy in acute respiratory failure. Respir Care. 2010:55:408-13.

9. Wagstaff TA, Soni N. Performance of six types of oxygen delivery devices at varying respiratory rates. Anaesthesia. 2007:62:492-503.

10. Corley A, Caruana LR, Barnett AG, et al. Oxygen delivery through high-flow nasal cannulae increase end-expiratory lung volume and reduce respiratory rate in post-cardiac surgical patients. Br J Anaesth. 2011;107:998-1004. 
11. Parke R, McGuinness S, Eccleston M. Nasal high-flow therapy delivers low level positive airway pressure. Br J Anaesth. 2009;103:886-90.

12. Maggiore SM, Idone FA, Vaschetto $R$, et al. Nasal high-flow versus Venturi mask oxygen therapy after extubation. Effects on oxygenation, comfort, and clinical outcome. Am J Respir Crit Care Med. 2014;190:282-8.

13. Rittayamai N, Tscheikuna J, Rujiwit P. High-flow nasal cannula versus conventional oxygen therapy after endotracheal extubation: a randomized crossover physiologic study. Respir Care. 2014;59:485-90.

14. Sztrymf B, Messika J, Mayot T, et al. Impact of high-flow nasal cannula oxygen therapy on intensive care unit patients with acute respiratory failure: a prospective observational study. J Crit Care. 2012;27:324.

15. Sztrymf B, Messika J, Bertrand F, et al. Beneficial effects of humidified high flow nasal oxygen in critical care patients: a prospective pilot study. Intensive Care Med. 2011;37:1780-6.

16. Schwabbauer N, Berg B, Blumenstock $G$, et al. Nasal high-flow oxygen therapy in patients with hypoxic respiratory failure: effect on functional and subjective respiratory parameters compared to conventional oxygen therapy and non-invasive ventilation (NIV). BMC Anesth. 2014;14:66.

17. Parke R, McGuinness S, Dixon R, et al. Open-label, phase II study of routine high-flow nasal oxygen therapy in cardiac surgical patients. Br J Anaesth. 2013;111:925-31.

18. Song HZ, Gu JX, Xiu HQ, Cui W, Zhang GS. The value of high-flow nasal cannula oxygen therapy after extubation in patients with acute respiratory failure. Clinics. 2017;72:562-7.

19. Corley A, Bull T, Spooner AJ, Barnett AG, Fraser JF. Direct extubation onto high-flow nasal cannulae post-cardiac surgery versus standard treatment in patients with a $\mathrm{BMI} \geq 30$ : a randomised controlled trial. Intensive Care Med. 2015:41:887-94

20. Moher D, Liberati A, Tetzlaff J, Altman DG, PRISMA Group. Preferred reporting items for systematic reviews and meta-analyses: the PRISMA statement. BMJ. 2009;339:b2535.

21. Higgins JPT, Green S. Cochrane handbook for systematic reviews of interventions. Version 5.1.0. Oxford: The Cochrane Collaboration; 2011. http://handbook-5-1.cochrane.org

22. Hernández G, Vaquero C, González P, Subira C, Frutos-Vivar F, Rialp G, et al. Effect of postextubation high-flow nasal cannula vs conventional oxygen therapy on reintubation in low-risk patients. JAMA. 2016;315:1354-61.

23. Fernandez R, Subira C, Frutos-Vivar F, Rialp G, Laborda C, Masclans JR, et al. High-fow nasal cannula to prevent postextubation respiratory failure in high-risk non-hypercapnic patients: a randomized multicenter trial. Ann Intensive Care. 2017;7:47

24. Guyatt G, Oxman AD, Akl EA, et al. GRADE guidelines: IntroductionGRADE evidence profiles and summary of findings tables. J Clin Epidemiol. 2011;64:383-94.

25. Tiruvoipati R, Lewis D, Haji K, Botha J. High-flow nasal oxygen vs high-flow face mask: a randomized crossover trial in extubated patients. J Crit Care. 2010;25:463-8.

26. Di mussi R, Spadaro S, Stripoli T, Volta CA, Trerotoli P, Pierucci P, et al. Highflow nasal cannula oxygen therapy decreases postextubation neuroventilatory drive and work of breathing in patients with chronic obstructive pulmonary disease. Crit Care. 2018;22:180

27. Futier E, Paugam-Burtz C, Godet T, Khoy-Ear L, Rozencwajg S, Delay JM, et al. Effect of early postextubation high-flow nasal cannula vs conventional oxygen therapy on hypoxaemia in patients after major abdominal surgery: a French multicentre randomised controlled trial (OPERA). Intensive Care Med. 2016:42:1888-98.

28. Parke RL, McGuinness SP. Pressures delivered by nasal high flow oxygen during all phases of the respiratory cycle. Respir Care. 2013;58:1621-4.

29. Nielsen KR, Ellington LE, Gray AJ, Stanberry LI, Smith LS, DiBlasi RM. Effect of high-flow nasal cannula on expiratory pressure and ventilation in infant, pediatric, and adult models. Respir Care. 2018;63(2):147-57.

30. Du X, Yang C, Pan P, Yu X. Effect of high-flow nasal cannula oxygen therapy on improving the atelectasis in adults after cardiac surgeries: a meta-analysis. Zhonghua Wei Zhong Bing Ji Jiu Yi Xue. 2018;30:748-53.

31. Delorme M, Bouchard PA, Simon M, Simard S, Lellouche F. Crit Care Med. 2017;45:1981-8.

32. Zhang JC, Wu FX, Meng LL, Zeng CY, Lu YQ. A study on the effects and safety of sequential humidified high flow nasal cannula oxygenation therapy on the COPD patients after extubation. Zhonghua Yi Xue Za Zhi. 2018;98(2):109-12.

33. Mauri T, Alban L, Turrini C, Cambiaghi B, Carlesso E, Taccone $P$, et al. Optimum support by high-flow nasal cannula in acute hypoxemic respiratory failure: effects of increasing flow rates. Intensive Care Med. 2017; 43:1453-63.

34. Roca O, Hernández G, Díaz-Lobato S, Carratalá JM, Gutiérrez RM, Masclans $J R$, et al. Current evidence for the effectiveness of heated and humidified high flow nasal cannula supportive therapy in adult patients with respiratory failure. Crit Care. 2016;20:109.

35. Dysart K, Miller T, Wolfson M, Shaffer T. Research in high flow therapy: mechanisms of action. Respir Med. 2009;103:1400-5.

36. Youfeng Z, Haiyan Y, Rui Z, Jianrui W. High-flow nasal cannula oxygen therapy versus conventional oxygen therapy in patients with acute respiratory failure: a systematic review and meta-analysis of randomized controlled trials. BMC Pulmonary Med. 2017;17:201.

37. Vincent $J$, Moreno R. Clinical review: Scoring systems in the critically ill. Crit Care. 2010;14:207

38. Poole D, Rossi C, Latronico N, Rossi G, Finazzi S, Bertolini G, et al. Comparison between SAPS II and SAPS 3 in predicting hospital mortality in a cohort of 103 Italian ICUs. Is new always better? Intensive Care Med. 2012; 38:1280-8.
Ready to submit your research? Choose BMC and benefit from:

- fast, convenient online submission

- thorough peer review by experienced researchers in your field

- rapid publication on acceptance

- support for research data, including large and complex data types

- gold Open Access which fosters wider collaboration and increased citations

- maximum visibility for your research: over $100 \mathrm{M}$ website views per year

At $\mathrm{BMC}$, research is always in progress.

Learn more biomedcentral.com/submissions 